\title{
Gestión de cuentas por cobrar y sus efectos en la liquidez en la facultad de una universidad particular, Lima, periodo 2010-2015.
}

\section{Management of accounts receivable and their effects on liquidity in the faculty of a private university, Lima, period 2010-2015.}

\author{
Gonzales Pisco Esmina; Sanabria Bolaños Sussan Wendy
}

\author{
EP. Contabilidad, Facultad de Ciencias Empresariales, Universidad Peruana Unión
}

\begin{abstract}
Resumen
El propósito de la investigación es determinar los efectos de las cuentas por cobrar en la liquidez en la facultad de una universidad particular, Lima, periodo 2010-2015. El nivel de estudio es descriptivo explicativo porque se establece el efecto que tiene la gestión de las cuentas por cobrar mediante los procedimiento de crédito, procedimiento de cobranza, ratios de actividad y morosidad en la liquidez que ha sido analizada mediante la liquidez corriente, razón ácida, razón de efectivo y capital de efectivo, planteándose la hipótesis general que la gestión de las cuentas por cobrar afecta en la liquidez en la facultad de una universidad particular, Lima 2010-2015. Del análisis de las respectivas variables de estudio tuvo como resultado que el nivel de significancia es un p-valor de 0,025 , menor a $\alpha=5 \%$ por lo cual se acepta la hipótesis alterna y se rechaza la hipótesis nula, es decir, los procedimientos de crédito afectan en el ratio de liquidez corriente, prueba ácida, razón de efectivo, capital de trabajo neto sobre el total de activos de la facultad de una universidad particular, Lima 2010-2015. En conclusión, según el estudio se concluye que la gestión de las cuentas por cobrar tiene un efecto sobre la liquidez de la facultad de una universidad particular, Lima, periodo 2010-2015. Es decir, cada una de las dimensiones de procedimientos de crédito, procedimiento de cobranzas, ratios de actividad y morosidad tiene una participación en la liquidez reflejado en los ratios de liquidez corriente, razón ácida, razón de efectivo y capital de trabajo neto sobre el total de activos.
\end{abstract}

Palabras Clave: Gestión de cuentas por cobrar y liquidez

\begin{abstract}
The purpose of the investigation is to determine the effects of the accounts receivable in the liquidity in the faculty of a private university, Lima, period 2010-2015. The level of study is explanatory descriptive because it establishes the effect of the management of accounts receivable through the credit procedure, collection procedure, activity ratios and delinquency in liquidity that has been analyzed through current liquidity, acid ratio, cash ratio and cash capital, considering the general hypothesis that the management of accounts receivable affects liquidity in the faculty of a private university, Lima 2010-2015. From the analysis of the respective study variables, it was found that the level of significance is a p-value of 0.025 , lower than $\alpha=5 \%$, for which the alternative hypothesis is accepted and the null hypothesis is rejected, that is, the procedures Credit affects the current liquidity ratio, acid test, cash ratio, net working capital over the total assets of the faculty of a private university, Lima 2010-2015. In conclusion, according to the study it is concluded that the management of accounts receivable has an effect on the liquidity of the faculty of a private university, Lima, period 2010-2015. That is, each one of the dimensions of credit procedures, collection procedure, activity ratios and delinquency has a participation in the liquidity reflected in the current liquidity, acid ratio, cash ratio and net working capital ratios over the total of assets.
\end{abstract}

Keywords: Management of accounts receivable and liquidity

\section{Introducción}

La gestión de cuentas por cobrar es un proceso donde se administra y gestiona las ventas al crédito (Rivera 2007). Para Merton y Bodie (citado por Lizárraga, 2010) es la extensión de un crédito comercial a los clientes por un tiempo prudencial; así mismo Bravo (citado por Lizárraga, 2010) indica que dicha gestión consiste en representar derechos exigibles originados por ventas, servicios prestados, otorgamientos de préstamos o cualquier otro concepto análogo; representan aplicaciones de recursos de la empresa que se transformarán en efectivo para terminar el ciclo financiero a corto plazo.

Después de conocer el concepto de gestión de cuentas por cobrar, Lizárraga (2010) menciona que es importante la aplicación de herramientas y técnicas adecuadas para su eficiente gestión, de forma que permitan lograr los objetivos y planes trazados durante un determinado período. Ettinger (2000) también destaca la importancia que tiene la gestión de cuentas por cobrar puesto que representan las pautas que regirán las condiciones sobre las cuales se otorgarán créditos a los clientes, para lograr con ello un control más eficiente sobre aquellos que cumplen puntualmente con las obligaciones contraídas. Así mismo en el estudio realizado por Noriega (citado por Aguilar, 2013) concluyó que es importante optimizar la gestión de cuentas por cobrar; aplicando políticas, procedimientos y períodos de crédito adecuados al giro del negocio, que proyecte beneficios económicos a las empresas; además de aplicar medidas de control y seguimiento a dichas políticas conforme las necesidades y evolución del negocio.

Autor de correspondencia:

E-mail:esmina.gonzales@gmail.com, suwen18@gmail.com 952813488,965461730 
Con respecto a la liquidez, Bernstein (citado por Aguilar, 2013) menciona que es la facilidad, velocidad y el grado de merma para convertir los activos circulantes en tesorería. También nos refiere como la posesión de la empresa de efectivo necesario en el momento oportuno que nos permita hacer el pago de los compromisos anteriormente contraídos (Mercados). Así mismo Van y Wachowicz (2002) también lo conceptualiza como la capacidad de un activo de convertirse en efectivo sin una concesión importante de precio. Por otro lado, Lawrence (2003) señala que liquidez es la capacidad de una empresa para satisfacer obligaciones a corto plazo conforme se venza. El análisis de liquidez de una empresa es especialmente importante para los acreedores; ya que, si una compañía tiene una posición de liquidez deficiente, puede generar un riesgo de crédito, y quizá producir incapacidad de efectuar pagos importantes y periódicos de interés. Monzón (2005) menciona que la liquidez dentro de las empresas es de suma importancia, esto se justifica por diferentes razones entre las que se hallan uno de los objetivos claves de la función financiera y es asegurar la solvencia dado que de esta deriva la posibilidad de alcanzar dos finalidades u objetivos básicos de la empresa: sobrevivir a largo plazo, si sobrevive conseguir su independencia o la autonomía siempre que estas se consideren valiosas por sí misma.

Según Joehnk (2005) los ratios de liquidez de especial importancia es si la empresa tiene una adecuada tesorería y otros activos líquidos a mano para satisfacer los pagos de la deuda y las necesidades operativas en tiempo y forma. Van y Wachowicz (2002) mencionan que las razones de liquidez se utilizan con el fin de determinar la capacidad de una empresa para cumplir con sus obligaciones a corto plazo. Comparan este tipo de obligaciones con los recursos disponibles a corto plazo (corrientes) con los que cuenta para cumplirlas, se destaca su importancia porque a partir de dichas razones se puede obtener bastante información acerca de la solvencia de las empresas y de su capacidad para seguir siendo solventes en caso de una adversidad.

Se tiene como referencia investigaciones realizadas por otros investigadores tal como Noriega (citado por Aguilar, 2013) titulado: "Administración de cuentas por cobrar, un enfoque para la toma de decisiones en la industria maquiladora de prendas de vestir en Guatemala" cuyo objetivo fue establecer procedimientos y políticas de crédito para la administración financiera de cuentas por cobrar en empresas que se dedican a la industria maquiladora de prendas de vestir. Del análisis efectuado, se concluyó que la tasa de rendimiento sobre la inversión para el año 2009 fue del $21.67 \%$, y el aporte de las ventas al crédito a este rendimiento fue del $15.78 \%$; dato significativo que demuestra que las cuentas por cobrar merecen especial atención. También se concluyó, a través de un estudio de escenarios factibles donde se calculan rubros importantes para optimizar la gestión de cuentas por cobrar; que es necesario aplicar políticas, procedimientos y períodos de crédito adecuados al giro del negocio, que proyecte beneficios económicos a las empresas.

Gonzales y Vera (2013) realizaron un estudio de campo de tipo descriptivo titulado "Incidencia de la Gestión de las cuentas por cobrar en la liquidez y Rentabilidad de la empresa Hierros San Félix, Guayana”. El propósito de esta investigación consistió en describir los procedimientos administrativos contables y determinar el efecto financiero de la gestión de cuentas por cobrar en la liquidez y rentabilidad. Del análisis efectuado concluyeron que por la demora de la cancelación de las facturas la empresa no tiene medidas de presión para los clientes morosos y que es necesario efectuar cobro de intereses moratorios.

Aguilar (2013) en su trabajo titulado: "Gestión de las cuentas por cobrar y su incidencia en la liquidez de la empresa contratista corporación petrolera SAC, Lima” realizó un estudio basado en el método deductivo de diseño no experimental, transaccional, cuyo objetivo fue determinar de qué manera la gestión de cuentas por cobrar incide en la liquidez llegando a su principal conclusión: la institución carece políticas de crédito eficientes, pues en la evaluación de los posibles clientes solo toma en cuenta el prestigio y la antigüedad en el mercado, más no se hace un estudio y seguimiento riguroso al comportamiento, que tienen los posibles clientes en el cumplimiento con sus obligaciones con otros contratistas.

Lizárraga (2010) realizó un trabajo de investigación titulado: "Gestión de las cuentas por cobrar en la administración del capital de trabajo de las empresas de fabricación de plásticos del Distrito de Ate", cuya investigación es de tipo aplicativo, de nivel descriptivo, explicativo, correlacional; siendo su objetivo principal establecer como una buena gestión de cuentas por cobrar impacta favorablemente en la administración de capital de trabajo. Se concluyó que sin una buena política de créditos no será posible que una empresa alcance niveles de cuentas por cobrar razonables que favorezcan la rotación de las cuentas por cobrar y la asignación correcta del crédito.

Se tiene como objetivo general determinar cuáles son los efectos de la gestión de las cuentas por cobrar en la liquidez en la facultad de una universidad particular, Lima 2010-2015. Por objetivos específicos describir los efectos del procedimiento de crédito en la liquidez durante los periodos 2014 - 2015. Determinar cuáles son los efectos de los procedimientos de crédito en el ratio de liquidez corriente, razón ácida, razón de efectivo, capital de trabajo neto sobre total de activos en la facultad de una universidad particular, Lima 2010-2015. Determinar cuáles son los efectos de los procedimientos de cobranza en el ratio de liquidez corriente, razón ácida, razón de efectivo, capital de trabajo neto sobre total de activos en la facultad de una universidad particular, Lima 20102015. Determinar cuáles son los efectos de los ratios de actividad en el ratio de liquidez corriente, razón ácida, razón de efectivo, capital de trabajo neto sobre total de activos en la facultad de una universidad particular, Lima 2010-2015.Determinar cuáles son los efectos de la morosidad en el ratio de liquidez corriente, razón ácida, razón de efectivo, capital de trabajo neto sobre total de activos en la facultad de una universidad particular, Lima 2010-2015.

\section{Método}

El tipo de investigación es cuantitativo con un alcance descriptivo explicativo. Cuantitativo porque mide las variables de estudio y aplica el análisis estadístico, y es explicativo, porque mediante el análisis de regresión lineal se identificará, analizará los efectos y las posibles repercusiones, de la gestión de cuentas por cobrar sobre la liquidez. Está desarrollado bajo un diseño no experimental, puesto que no pretende manipular deliberadamente ninguna de las variables. El tipo del trabajo es retrospectivo y longitudinal porque se evaluaron datos correspondientes a 6 años (2010-2015). Es causa-efecto porque pretende conocer los efectos 
de la gestión de cuentas por cobrar en la liquidez. Los datos de la empresa que fue analizada pertenecen a los estudiantes de la facultad de una universidad particular con una promedio de 1100 estudiantes desde el año 2010 al 2012, facultad que cuenta con 5 escuelas académicas, donde se tomó como referencia solo a los estudiantes con deudas pendientes tanto al inicio de un ciclo académico, durante y al final del ciclo académico, también se encuentra los saldos deudores de los estudiantes que incurrieron en mora por incumplimiento de sus obligaciones de pago. Las variables de estudio son: gestión de cuentas por cobrar cuyas dimensiones son procedimiento de crédito, procedimiento de cobranza, ratios de actividad y morosidad y como segunda variable está la liquidez cuyos indicadores son liquidez corriente, prueba ácida, razón de efectivo y capital neto sobre el total de activos.

Los datos fueron tomados del informe financiero contable proporcionados por la facultad de la universidad particular desde el año 2010 hasta el año 2015, periodo de 6 años en cortes de 6 meses, cuya elección se realizó de manera no probabilística. Se utilizó el programa estadístico informático SPSS 22, estadística paramétrica con regresión lineal simple coeficiente de determinación, análisis de correlación para obtener el P valor y el análisis de estadística descriptiva de tablas frecuentes. Así mismo si $p \geq 0.05$ se acepta la hipótesis nula, si $p \leq 0.05$ se rechaza la hipótesis alterna donde la hipótesis nula viene a ser: Los procedimientos de crédito no afectan en la liquidez: ratios de liquidez corriente, razón ácida, razón de efectivo, capital de trabajo neto sobre total de activos en la facultad de una universidad particular, Lima 2010-2015. Y la hipótesis alterna es: Los procedimientos de crédito no afectan en la liquidez: ratios de liquidez corriente, razón ácida, razón de efectivo, capital de trabajo neto sobre total de activos en la facultad de una universidad particular, Lima 2010-2015.

\section{Resultados}

Los resultados consisten en el análisis de la Gestión de cuentas por cobrar y liquidez; efecto de los procedimiento de crédito en el ratio de liquidez corriente, razón acida, razón de efectivo, capital del trabajo neto sobre el total de activos; efecto de los procedimiento de cobranza en el ratio de liquidez corriente, razón acida, razón de efectivo, capital del trabajo neto sobre el total de activos; efecto de los ratios de actividad en el ratio de liquidez corriente, razón acida, razón de efectivo, capital del trabajo neto sobre el total de activos y efectos de la morosidad en el ratio de liquidez corriente, razón acida, razón de efectivo, capital del trabajo neto sobre el total de activos.

En la Tabla 1 según el procedimiento de crédito se observa un mayor efecto (participación) capital de trabajo neto sobre total de activos con un $71.00 \%$, mientras que en la evaluación del crédito existe una igualdad de participación en la liquidez corriente y razón ácida con un 49.00\%; a diferencia en la razón de efectivo un $41.00 \%$. Respecto al análisis de riesgo se observa una mayor participación en el capital de trabajo neto sobre total de activos a un $52.00 \%$, en tanto que, en la liquidez corriente y en la razón ácida son afectadas en igual porcentaje $49.00 \%$, por último, en la razón de efectivo $40.00 \%$. El análisis estadístico arrojo un nivel de significancia p-valor de 0,025 , menor a $\alpha=5 \%$ por lo cual se acepta la hipótesis alterna y se rechaza la hipótesis nula, es decir, los procedimientos de crédito afectan en el ratio de liquidez corriente, prueba ácida, razón de efectivo, capital de trabajo neto sobre el total de activos en la facultad de una universidad particular, Lima 2010-2015.

Tabla 1.

Efectos de procedimiento de crédito, en la liquidez corriente, prueba ácida, razon de efectivo y capital de trabajo neto sobre el total de activos.

\begin{tabular}{|c|c|c|c|c|c|c|c|c|c|c|c|c|}
\hline & \multicolumn{4}{|c|}{ Liquidez corriente } & \multicolumn{2}{|l|}{$\begin{array}{l}\text { Razón } \\
\text { Ácida }\end{array}$} & \multicolumn{2}{|c|}{$\begin{array}{l}\text { Razón de } \\
\text { Efectivo }\end{array}$} & & \multicolumn{3}{|c|}{$\begin{array}{l}\text { Capital de trabajo neto } \\
\text { sobre total de activos }\end{array}$} \\
\hline de Crédito & $\mathrm{R}$ & $\mathrm{R}^{2}$ & $\mathrm{P}$ & $\mathrm{R}$ & $\mathrm{R}^{2}$ & $\mathrm{p}$ & $\mathrm{R}$ & $\mathrm{R}^{2}$ & $\mathrm{p}$ & $\mathrm{R}$ & $\mathrm{R}^{2}$ & $\mathrm{p}$ \\
\hline $\begin{array}{l}\text { Evaluación del } \\
\text { Crédito } \\
\text { Análisis de }\end{array}$ &,$- 700 *$ & 0.49 & 0.011 &,$- 699 *$ & 0.49 & 0.011 &,$- 643 *$ & 0.41 & 0.01 &,$- 843 * *$ & 0.71 & 0.00 \\
\hline Riesgo &,$- 702 *$ & 0.49 & 0.011 &,$- 701 *$ & 0.49 & 0.011 &,$- 635^{*}$ & 0.40 & 0.01 &,$- 722 * *$ & 0.52 & 0.01 \\
\hline
\end{tabular}

**. La correlación es significativa en el nivel 0,01 (2 colas).

*. La correlación es significativa en el nivel 0,05 (2 colas).

En la tabla 2 según el procedimiento de cobranza se observa que la condición de cobranza cinco armadas existen una igualdad teniendo mayor participación con un $69.00 \%$ en la liquidez corriente y razón ácida, a diferencia la razón de efectivo afecta con un $61.00 \%$ y en el capital de trabajo neto sobre total de activos $62.00 \%$. Referente a los medios de cobranza, el medio de bancos tiene mayor participación con un $64.00 \%$ sobre el capital de trabajo neto, en tanto que en la liquidez corriente $56.00 \%$, razón ácida $56.00 \%$ y razón de efectivo $59.00 \%$. El análisis estadístico arrojo un nivel de significancia p-valor de 0,015 menor a $\alpha=5 \%$ por lo cual se rechaza la hipótesis nula y aceptamos la hipótesis alterna es decir los procedimientos de cobranza afectan en el ratio de liquidez corriente, razón ácida, razón de efectivo, capital de trabajo neto sobre total de activos en la facultad de una universidad particular, Lima 2010-2015. 
Tabla 2.

Efectos de procedimiento de cobranza en la liquidez corriente, prueba acida, razón de efectivo y capital de trabajo neto sobre el total de activos

\begin{tabular}{|c|c|c|c|c|c|c|c|c|c|c|c|c|}
\hline \multirow[b]{2}{*}{$\begin{array}{l}\text { Procedimiento } \\
\text { de Cobranza }\end{array}$} & \multicolumn{3}{|c|}{ Liquidez corriente } & \multicolumn{3}{|c|}{$\begin{array}{l}\text { Razón } \\
\text { Ácida }\end{array}$} & \multicolumn{2}{|c|}{$\begin{array}{l}\text { Razón de } \\
\text { Efectivo }\end{array}$} & \multicolumn{4}{|c|}{$\begin{array}{c}\text { Capital de trabajo neto } \\
\text { sobre total de activos }\end{array}$} \\
\hline & $\mathrm{R}$ & $\mathrm{R}^{2}$ & $\mathrm{P}$ & $\mathrm{R}$ & $\mathrm{R}^{2}$ & $\mathrm{p}$ & $\mathrm{R}$ & $\mathrm{R}^{2}$ & $\mathrm{p}$ & $\mathrm{R}$ & $\mathrm{R}^{2}$ & $\mathrm{p}$ \\
\hline Contado & -.484 & 0.23 & .111 & -.482 & 0.23 & .113 & -.387 & 0.15 & 214 & -.519 & 0.27 & .084 \\
\hline Dos Armadas &,$- 613^{*}$ & 0.38 & .034 &,$- 612^{*}$ & 0.37 & .035 & $\begin{array}{c}-, 520 \\
-\end{array}$ & 0.27 & 083 & -.574 & 0.33 & .051 \\
\hline Cinco Armadas &,$- 829 * *$ & 0.69 & .001 &,$- 829 * *$ & 0.69 & .001 & ,778** & 0.61 & 003 &,$- 790 * *$ & 0.62 & .002 \\
\hline Caja &,$- 757 * *$ & 0.57 & .004 &,$- 756 * *$ & 0.57 & .004 &,$- 688^{*}$ & 0.47 & 013 &,$- 805^{* *}$ & 0.65 & .002 \\
\hline Bancos &,$- 747 * *$ & 0.56 & .005 &,$- 746 * *$ & 0.56 & .005 &,$- 673 *$ & 0.45 & 016 &,$- 799 * *$ & 0.64 & .002 \\
\hline
\end{tabular}

**. La correlación es significativa en el nivel 0,01 (2 colas).

*. La correlación es significativa en el nivel 0,05 (2 colas).

En la tabla 3 muestra según el ratio de cuentas por cobrar se observa una igualdad de mayor participación en la liquidez corriente y razón ácida con un $44.00 \%$, a diferencia la razón de efectivo $40.00 \%$ y finalmente el capital de trabajo neto sobre total de activos representa un $18.00 \%$. Respecto al plazo promedio de cobro se muestra que tiene mayor efecto la razón de efectivo con un 5\%, a diferencia que, en la liquidez corriente y razón ácida tienen un efecto de 3\% respectivamente.

El análisis estadístico arrojo un nivel de significancia p-valor de 0,065 , es mayor a $\alpha=5 \%$ por lo cual se acepta la hipótesis nula y se rechaza la hipótesis alterna, es decir, los ratios de actividad no afectan en el ratio de liquidez corriente, razón ácida, razón de efectivo, capital de trabajo neto sobre total de activos en la facultad de una universidad particular, Lima 2010-2015.

Tabla 3 Efectos de ratios de actividad en la liquidez corriente, razón ácida, razón de efectivo y capital de trabajo neto sobre total de activos.

\begin{tabular}{|c|c|c|c|c|c|c|c|c|c|c|c|c|}
\hline \multirow{2}{*}{ Ratios de Actividad } & \multicolumn{2}{|c|}{$\begin{array}{l}\text { Liquidez } \\
\text { corriente }\end{array}$} & & \multicolumn{3}{|c|}{$\begin{array}{l}\text { Razón } \\
\text { Ácida }\end{array}$} & \multicolumn{3}{|c|}{ Razón de Efectivo } & \multicolumn{3}{|c|}{$\begin{array}{c}\text { Capital de trabajo neto } \\
\text { sobre total de activos }\end{array}$} \\
\hline & $\mathrm{R}$ & $\mathrm{R}^{2}$ & $\mathrm{p}$ & $\mathrm{R}$ & $\mathrm{R}^{2}$ & $\mathrm{p}$ & $\mathrm{R}$ & $\mathrm{R}^{2}$ & $\mathrm{p}$ & $\mathrm{R}$ & $\mathrm{R}^{2}$ & $\mathrm{p}$ \\
\hline $\begin{array}{l}\text { Ratio cuentas por } \\
\text { cobrar }\end{array}$ &,$- 664 *$ & 0.44 & 019 & , $663^{-}$ & 0.44 & .019 & , $636 *$ & 0.40 & .026 & .427 & 0.18 & .166 \\
\hline $\begin{array}{l}\text { Plazo promedio de } \\
\text { cobro }\end{array}$ & .170 & 0.03 & 598 & .169 & 0.03 & .599 & .224 & 0.05 & .483 & $\begin{array}{c}- \\
.021\end{array}$ & 0.00 & .949 \\
\hline
\end{tabular}

**. La correlación es significativa en el nivel 0,01 (2 colas).

*. La correlación es significativa en el nivel 0,05 (2 colas).

En la tabla 4 se observa según el nivel de morosidad se observa una mayor participación del capital de trabajo neto sobre total de activos en un $19.00 \%$, mientras que, existe una igualdad en la liquidez corriente y razón ácida del $10.00 \%$; a diferencia que, la razón de efectivo se observa un $6 \%$. Referente a la tasa de interés moratorio se muestra un mayor efecto en el capital de trabajo neto sobre total de activos en un 6\%; mientras que, existe una igualdad de efecto en la liquidez corriente y razón ácida del 4\%; a diferencia de la razón de efectivo se observa un $5 \%$.

El análisis estadístico arrojo nivel de significancia es un p- valor de 0,310 es mayor a $\alpha=5 \%$ por lo cual aceptamos la hipótesis nula y rechazamos la hipótesis alterna, es decir, la morosidad no afecta en el ratio de liquidez corriente, razón ácida, razón de efectivo, capital de trabajo neto sobre total de activos en la facultad de una universidad particular, Lima 2010-2015. 
Efectos de morosidad en la liquidez corriente, razón ácida, razón de efectivo, capital de Trabajo neto sobre notal de activos.

\begin{tabular}{|c|c|c|c|c|c|c|c|c|c|c|c|c|}
\hline \multirow[t]{2}{*}{ Morosidad } & \multicolumn{3}{|c|}{ Liquidez corriente } & \multicolumn{2}{|c|}{$\begin{array}{l}\text { Razón } \\
\text { Ácida }\end{array}$} & \multicolumn{4}{|c|}{ Razón de Efectivo } & \multicolumn{3}{|c|}{$\begin{array}{c}\text { Capital de Trabajo } \\
\text { Neto sobre total de } \\
\text { Activos }\end{array}$} \\
\hline & $\mathrm{R}$ & $\begin{array}{c}\mathrm{R} \\
\text { cuadrado }\end{array}$ & $\mathrm{P}$ & $\mathrm{R}$ & $\begin{array}{c}\mathrm{R} \\
\text { cuadrado }\end{array}$ & $\mathrm{p}$ & $\mathrm{R}$ & $\begin{array}{c}\mathrm{R} \\
\text { cuadrado }\end{array}$ & $\mathrm{P}$ & $\mathrm{R}$ & $\begin{array}{c}\mathrm{R} \\
\text { cuadrado }\end{array}$ & $\mathrm{p}$ \\
\hline \multirow{2}{*}{$\begin{array}{l}\text { Nivel de Morosidad } \\
\text { Tasa de Interés Moratorio }\end{array}$} & .322 & 0.10 & .307 & .321 & 0.10 & .308 & .241 & 0.06 & .451 & .436 & 0.19 & .157 \\
\hline & -.199 & 0.04 & .535 & -.199 & 0.04 & .534 & -.222 & 0.05 & .487 & -.248 & 0.06 & .437 \\
\hline
\end{tabular}

**. La correlación es significativa en el nivel 0,01 (2 colas).

\section{Discusión}

Los resultados que se obtuvieron en esta investigación, permitieron comprobar las hipótesis planteadas en el primer capítulo del presente proyecto investigativo; prueba de ello lo constituyen los procedimientos que se aplicaron para determinar la incidencia de la gestión de las cuentas por cobrar en la liquidez en la facultad de una universidad particular periodos 2010-2015; la comprobación de las hipótesis, está en consonancia con lo que expone la autor: Víctor Humberto Aguilar Pinedo del trabajo investigativo titulado: "La gestión de las cuentas por cobrar y su incidencia en administración del capital de trabajo de las empresas de fabricación de plástico del distrito de Ate", quien llega a las siguientes conclusión: Sin una buena política de créditos no será posible que una empresa alcance niveles de cuentas por cobrar razonables que favorezcan la rotación de las cuentas por cobrar. Con respecto a la liquidez se resume diciendo que sin manejo de liquidez no hay empresa, porque si no hay dinero o clientes que deban, cómo se podrá cumplir con las obligaciones o deudas. Quien también llegó a la conclusión La administración eficiente de cuentas por cobrar en una empresa dedicada a la industria maquiladora de prendas de vestir, depende de la planificación e implementación de procedimientos y políticas de crédito acorde del giro del negocio, las cuales deben ser cumplidas a cabalidad, además de ser revisadas y controladas periódicamente para asegurar una óptima gestión administrativa de cuentas por cobrar.

En presente trabajo de investigación también se concluyó, respecto a los procedimientos de crédito, la evaluación del crédito, en el largo plazo, tiene una mayor participación o efecto en el capital de trabajo neto sobre el total de activos. Es decir, el nivel de activo circulante es adecuado a pesar de existir cuentas por cobrar originadas al inicio de las matrículas, por las tardanzas de los estudiantes en cancelar sus obligaciones financieras. Estos resultados también concuerdan de manera contraria pero dando el mismo énfasis a pesar de no ser un empresa en el rubro educativo con la investigación. Gestión de cuentas por cobrar y su incidencia en la liquidez de la empresa contratista corporación petrolera S.A.C. - año 2012 donde se detalla que la Corporación Petrolera SAC, cuenta con un capital de trabajo negativo para el año 2012, de - S/ 15’075,041 debido a la demora en la gestión de las cuentas por cobrar lo que no le permite contar con una liquidez necesaria para cubrir las necesidades de capital de trabajo.

Respecto al procedimiento de cobranza, la condición de cinco armadas tiene mayor participación o efecto sobre el ratio de liquidez corriente, debido a que la mayoría de estudiantes se acogen a esta modalidad de cobranza; confirmada con el ratio razón de efectivo que refleja el importe de las cuentas caja y bancos; la mayor cantidad de estudiantes se acogen a esta modalidad por la facilidad de pago, quien también lo afirma es la investigación Trabajo sobre procedimientos convenidos en las áreas de los ingresos y cuentas por cobrar de un colegio educativo privado de enseñanza media presentado por Nelson Everaldo Ixcoy Equité (2012): los ingresos de un colegio educativo privado de enseñanza media en Guatemala pueden ser controladas de manera práctica, debido a que estos son cuotas autorizados por ministerio de educación de acuerdo al entorno del colegio y además existe un contrato de servicios educativos autorizado previamente por la dirección de atención y asistencia, en el cual se fijan las cuotas de inscripción y colegiaturas autorizadas por el periodo de año.

Respecto a la morosidad, en el nivel de morosidad, se observa que tiene un mayor efecto en el capital de trabajo neto sobre total de activo, en el largo plazo. Es decir, en el nivel de activo se encuentra las deudas totales (deudas inicio matricula y durante el ciclo académico).

Esto coincide con una investigación: en este último caso, cuando el proveedor cobra con antelación al plazo legal (asumiendo la entidad de crédito el riesgo de insolvencia), se produce un cambio en la titularidad del crédito que pasa de un proveedor a la entidad de crédito, declarándose así a la central de información de riesgos del Banco de España, lo cual constituye endeudamiento a corto o largo plazo del sector público.

\section{Conclusiones}

Después de realizar el análisis, se concluye que la gestión de las cuentas por cobrar tiene un efecto sobre la liquidez en la facultad de una Universidad particular, Lima, periodo 2010 - 2015. Es decir, cada una de las dimensiones de procedimientos de crédito, procedimiento de cobranzas, ratios de actividad y morosidad tiene una participación en la liquidez reflejado en los ratios de liquidez corriente, razón ácida, razón de efectivo y capital de trabajo neto sobre el total de activos.

Respecto a los procedimientos de crédito, la evaluación del crédito, en el largo plazo, tiene una mayor participación o efecto en el capital de trabajo neto sobre el total de activos. Es decir, el nivel de activo circulante es adecuado a pesar de existir cuentas por cobrar originadas al inicio de las matrículas, por las tardanzas de los estudiantes en cancelar sus obligaciones 
financieras. Lo mismo refleja el análisis de riesgo que presenta un efecto en el capital de trabajo neto sobre el total de activos. Es decir, el nivel de activo circulante es adecuado a pesar de existir cuentas por cobrar originadas al cierre de las matrículas, por las tardanzas de los estudiantes en cancelar sus obligaciones financieras.

Respecto al procedimiento de cobranza, la condición de cinco armadas tiene mayor participación o efecto sobre el ratio de liquidez corriente, debido a que la mayoría de estudiantes se acogen a esta modalidad de cobranza; confirmada con el ratio razón de efectivo que refleja el importe de las cuentas caja y bancos.

En cuanto a los ratios de actividad se observa una mayor participación en la liquidez corriente, debido a que según las veces de rotación de nuestras cuentas por cobrar se ha logrado la recaudación del efectivo quedando aún cuentas por cobrar. Mientras que en el ratio de efectivo según las veces de rotación de las cuentas por cobrar se ha logrado la recaudación en el efectivo.

Respecto a la morosidad, en el nivel de morosidad, se observa que tiene un mayor efecto en el capital de trabajo neto sobre total de activo, en el largo plazo. Es decir, en el nivel de activo se encuentra las deudas totales (deudas inicio matricula y durante el ciclo académico).

\section{Referencias}

Aguilar, V. (2013). Gestión de cuentas por cobrar y su incidencia en la liquidez de la empresa contratista corporación petrolera s.a.c. - año 2012. Universidad San Martín de Porres. Retrieved from http://www.repositorioacademico.usmp.edu.pe/handle/usmp/653

Aldas, I., \& Yánez, D. (2014). Análisis del rubro cuentas por cobrar y su efecto en la rentabilidad de la empresa J.M. Palau Agencia de vapores CIA LTDA. Universidad Técnica de Babahoyo. Retrieved from http://dspace.utb.edu.ec/handle/49000/793

Castillo, Y. (2012). Evaluación financiera de la liquidez en las empresas distribuidoras de productos farmaceúticos a través del ciclo de conversión de efectivo. Universidad de San Carlos de Guatemala.

Castro, P. (2013). El sistema de detracciones del IGV y su impacto en la liquidez de la empresa de transportes de carga pesada Factoria comercial y transportes S.A.C. Universidad privada Antenor Orrego.

Díaz Llanes, M. (2012). Inmovilizado: El punto de partida de la liquidez. Revista Universo Contábil, $134-145$. http://doi.org/10.4270/ruc.2012217

Gonzales, A., \& Vera, E. (2013). Incidencia de la gestion de las cuentas por cobrar en la liquidez y rentabilidad de la empresa Hierros San Felix CA. Universidad Nacional Experimental de Guayana. Retrieved from http://www.cidar.uneg.edu.ve/DB/bcuneg/EDOCS/TESIS/TESIS_PREGRADO/PROY/PROY046420 13GonzalezVera.pdf

González, J., \& Gomes, R. (2012). La Morosidad; un acuciante problema financicero de nuestros días. Saudi Med J, 33, 3-8. http://doi.org/10.1073/pnas.0703993104

Guajardo, G., \& Andrade, N. (2005). Contabilidad financiera. (E. McGraw-Hill, Ed.), Contabilidad financiera (5ta Edició).

Lizarraga, K. (2010). Gestión de las cuentas por cobrar en la administración del capital de trabajo de las empresas de fabricación de plástico del distrito de ate. Universidad San Martín de Porres. Retrieved from http://www.repositorioacademico.usmp.edu.pe/bitstream/usmp/360/1/lizarraga_ke.pdf

Mercados, M., Idec, F., Fabra, P., Velat, T., Pa, R., \& Arias, A. O. (n.d.). La Gestión de la Liquidez. Retrieved from http://www.barcelonaschoolofmanagement.upf.edu/documents/Tesina-La-gestion-de- la-liquidez.pdf

Noriega, J. (2011). Administración de cuentas por cobrar-un enfoque para la toma de decisiones en la industria maquiladora de prendas de vestir en Guatemala. Universidad de San Carlos de Guatemala.

Oto, B. (2011). Modelo de gestión financiera y reducción de morosidad en el departamento financiero de la Empresa Eléctrica Riobamba S.A.

Parrales, C. (2013). Análisis del índice de morosidad en la cartera de créditos del IECE-Guayaquil y propuesta de mecanismos de prevención de morosidad y técnicas eficientes de cobranzas. Universidad Politécnica Salesiana, sede Guayaquil.

Rangel, M. (2009). Gestión de las cuentas por cobrar y su efecto en la rentabilidad de las empresas comerciales del Municipio Valera. Journal of Chemical Information and Modeling. Universidad de Los Andes Rafael Rangel.

Rivero, A. (2014). Propuesta de estrategia financiera para el departamento de crédito y cobranzas para el mejoramiento de la gestión de cobranzas, caso en la empresa venezolana del vidrio. Universidad de Carabobo. Retrieved from http://mriuc.bc.uc.edu.ve/bitstream/handle/123456789/1233/arivero.pdf?sequence=1

Uceda, L., \& Villacorta, F. (2014). Las políticas de ventas al crédito y su influencia en la morosidad de los clientes de la empresa Import Export Yomar E.I.R.L. Universidad privada Antenor Orrego. Retrieved from http://www.gonzalezcabeza.com/documentos/CRECIMIENTO_MICROBIANO.pdf

Vallado, R. (2010). Administración financiera de cuentas por cobrar. Retrieved from http://es.slideshare.net/jcfdezmx2/cuentas-por-cobrar-presentation

Wittlinger, Carranza, \& Mori. (2008). Mejores Prácticas en Estrategias de Cobranza, 26, 1-23. Retrieved from http://www.smartcampaign.org/storage/documents/Tools_and_Resources/IS26SP.pdf 